\title{
Nursing Activities Score: Comparação com o Índice APACHE II e a Mortalidade em Pacientes Admitidos em Unidade de Terapia Intensiva*
}

\author{
Nursing Activities Score: Comparison among the Index APACHE II \\ and the Mortality in Patients Admitted in Intensive Care Unit
}

\author{
Lilia de Souza Nogueira', Michelle Rocha Santos², Sérgio Elia Mataloun ${ }^{3}$, Marcelo Moock ${ }^{4}$
}

\section{RESUMO}

JUSTIFICATIVA E OBJETIVOS: Não existem estudos que correlacionem o Nursing Activities Score (NAS), índice que determina o tempo real de avaliação da duração das atividades da enfermagem, com mortalidade e índices de gravidade. Os objetivos deste estudo foram conhecer os valores do NAS na unidade, verificar se há correlação desses valores com o APACHE II e analisar a mortalidade conforme os escores do NAS.

MÉTODO: Estudo prospectivo de pacientes internados na UTI no período de julho a novembro de 2005. Os dados referentes ao APACHE II foram recuperados pelo Sistema QuaTI. O teste do Qui-quadrado ou equivalente foi utilizado para comparação das proporções. Para os cálculos utilizou-se o EPI INFO-6.

RESULTADOS: A amostra foi de 148 pacientes com

1. Enfermeira da UTI do Hospital Estadual do Grajaú (HEG), Especialista em Terapia Intensiva e Cardiologia, Mestranda pela Escola de Enfermagem da Universidade de São Paulo.

2. Enfermeira do Hospital Israelita Albert Einstein, Especialista em Terapia Intensiva e Transplantes.

3. Médico Assistente Diarista da UTI do HEG, Professor Adjunto da Faculdade de Medicina / Universidade de Santo Amaro (UNISA).

4. Médico Responsável Técnico da UTI do HEG, Chefe da Disciplina de Medicina Intensiva da Faculdade de Medicina / UNISA.

*Recebido do Hospital Estadual de Grajaú (HEG), São Paulo, SP

Apresentado em 11 de janeiro de 2007

Aceito para publicação em 28 de agosto de 2007

Endereço para correspondência:

Lilia de Souza Nogueira

Rua Engenheiro José Salles, 200 Bloco 5/ Apt ${ }^{\circ} 111$

Interlagos

04776-100 - São Paulo, SP

Fones: (11) 5667-3399 / (11) 9131-0447

E-mail: lilianogueira@usp.br

(C)Associação de Medicina Intensiva Brasileira, 2007 mediana de idade de 55,5 anos, à razão de 59,4\% para o sexo masculino, tempo médio de permanência de 9,1 dias, taxa de mortalidade de $29,7 \%$, escore médio do NAS de $51,5 \%$ e APACHE II médio de 13,4 . Houve correlação positiva entre os valores do NAS e do APACHE II $(R=0,82)$. Separando os pacientes com NAS > 51 observou-se que 41/83 não sobreviveram e 42/83 sobreviveram; já naqueles com NAS < 51, 3/65 não sobreviveram e 62/65 sobreviveram ( $p<0,005)$, identificando-se diferença estatística significativa entre os grupos.

CONCLUSÕES: Nesta série o valor médio do NAS foi de $51,5 \%$. Houve boa correlação com o APACHE II e identificou-se que a mortalidade foi maior nos pacientes com valores do NAS mais elevados.

Unitermos: Enfermagem, Índice de gravidade de doença, Mortalidade, Unidade de Terapia Intensiva.

\section{SUMMARY}

BACKGROUND AND OBJECTIVES: Searching the literature, we found no studies which correlate the NAS (Nursing Activities Score), who determines the real time of nursing evaluation and patient care, with mortality for prognostic index. The objectives this study were to know the values of the NAS score at ours service, try to find correlations between this values and the APACHE II index and to analyze the mortality rates with NAS scores.

METHODS: This is a prospective ICU inpatient study from July to November/2005. Our data's of the APACHE II score were recorded from the QuaTI system study. Qui-square test or equivalent was done to compare the proportions. For the analyses we utilized the EPIINFO-6 software.

RESULTS: The sample was 148 patients, mean age of 55.5 years with $59.4 \%$ males. The mean hospitalization time were 9.1 days, mortality rate of $29.7 \%$, mean NAS 
score of $51.5 \%$ and mean APACHE II score of 13.4 . There were a positive correlation ship between NAS and APACHE II index $(R=0.82)$. Selecting only the patients with NAS more than 51 we found that 41 in 83 died and 42 in 83 survived. Those whose NAS were under 51, only 3 in 65 died and 62 in 65 survived ( $p<$ $0.005)$, identifying an statistically significant group.

CONCLUSIONS: In this study the mean value of NAS were $51.5 \%$. There were good correlation with the APA$\mathrm{CHE}$ II index and we shown that the mortality rate was high in the higher NAS values.

Key Words: Intensive Care Unit, Mortality, nursing, Severity of illness index.

\section{INTRODUÇÃO}

Os índices de gravidade têm como objetivo principal a descrição quantitativa do grau de disfunção orgânica de pacientes gravemente enfermos, cuja gravidade é traduzida em valor numérico a partir das alterações clínicas e laboratoriais existentes ou do tipo/número de procedimentos utilizados, permitindo a avaliação de desempenho da unidade de terapia intensiva (UTI) e da eficiência do tratamento realizado'. Entre os métodos mais utilizados na avaliação dos pacientes de UTI, encontram-se sistemas padronizados e aceitos internacionalmente pela comunidade científica para predição de mortalidade, como o Acute Physiology and Chronic Health Evalution (APACHE) ${ }^{2}$. Desenvolvido em 1981 por Knaus e col. esse índice, em 1985, foi submetido a uma revisão e simplificação, sendo denominado APACHE II. Para seu cálculo, habitualmente aplicado nas primeiras 24 horas de internação na UTI, são utilizadas 12 variáveis clínicas, fisiológicas e laboratoriais padronizadas, pontuadas de zero a quatro, conforme o grau de desvio da normalidade apresentado; além disso, pontos são atribuídos à idade, presença de doença crônica e variáveis fisiológicas, cuja soma fornece uma pontuação final que permite calcular o risco de óbito para o paciente ${ }^{3}$. Portanto, as avaliações de desempenho assistencial através da utilização de indicadores prognósticos permitem confrontar a mortalidade esperada com a observada, propiciando a análise seqüencial da unidade ao longo do tempo, além da comparação externa com outras unidades semeIhantes. Paralelamente à criação dos indicadores de gravidade, dentro do contexto da enfermagem, houve uma busca por índices que permitissem categorizar os pacientes segundo o grau de complexidade da assistência, permitindo mensurar o seu nível de gravidade e a correspondente carga de trabalho da enfermagem dispensada. Dentre os índices mais utilizados mundialmente, destaca-se o Therapeutic Intervention Scoring System (TISS), criado por Cullen e col. em 19744. Após ser submetido a diversas revisões e atualizações, em 1996, Miranda e col. publicaram a mais recente simplificação, denominada TISS-28, no qual 28 intervenções de enfermagem são analisadas e submetidas a pontuações, cujo escore final pode variar de 0 a 76 pontos. Esse indicador permite estimar quantas intervenções de enfermagem foram realizadas no paciente, sua gravidade, além de dimensionar a carga de trabalho da enfermagem, uma vez que cada ponto do TISS-28 consome 10,6 min do tempo de um enfermeiro na assistência direta ao paciente ${ }^{5}$. Em 2003, Miranda e col. constataram que o TISS-28 não refletia adequadamente a carga de trabalho dispensada ao paciente. Propôs então o desenvolvimento de outro indicador, o Nursing Activities Score (NAS), com a finalidade de estimar, com precisão, a carga de trabalho necessária. Esse índice analisa 23 intervenções assistenciais, pondera estas atividades e consolida um valor final que indica o tempo real da duração das atividades da enfermagem para cada paciente, analisado por turno de trabalho. Esse valor pode variar de $0 \%$ a $177 \%{ }^{6}$. Traduzido e validado no Brasil em 2002 por Queijo ${ }^{7}$, o NAS tornou-se um instrumento mais abrangente no sentido de medir a real carga de trabalho de enfermagem na UTI. Conforme a publicação original, o NAS foi desenvolvido como preditor de carga de trabalho de enfermagem, independente da gravidade do paciente. Há, contudo, uma percepção que pacientes mais graves demandam mais carga de trabalho. Para aferir esta hipótese, resolveu-se estudar a relação entre os escores do NAS com aqueles obtidos através do APACHE II, como perspectiva de encontrar respostas entre a existência ou não de correlação de gravidade e mortalidade com carga de trabalho de enfermagem.

Os objetivos deste estudo foram conhecer os valores do NAS na UTI, verificar se há correlação desses valores com o APACHE II e analisar a mortalidade conforme os escores do NAS.

\section{MÉTODO}

O presente estudo foi realizado na UTI-Adulto do Hospital Estadual do Grajaú, após aprovação do Comitê de Ética e Pesquisa da instituição. A unidade possui 10 leitos, atendendo todas as especialidades. Com caráter prospectivo, exploratório, descritivo e de abordagem 
quantitativa, foram analisados os pacientes internados na unidade no período de julho a novembro de 2005, que responderam aos critérios de inclusão (idade igual ou superior a 12 anos e permanência mínima de 24 horas na unidade). Através de um instrumento de coleta de dados, foram analisadas as variáveis demográficas (idade, sexo, procedência e tempo de internação), além do motivo de sua internação, de acordo com o CID-10 e o destino do paciente (alta, óbito ou transferência). Os valores do NAS foram obtidos através da aplicação do instrumento traduzido com o auxílio das anotações de enfermagem, prescrição médica e balanço hídrico do paciente. O APACHE II foi recuperado pelo sistema de Qualidade em Terapia Intensiva (QuaTI), um aplicativo desenvolvido pela DIXTAL e Associação de Medicina Intensiva Brasileira - AMIB, que permite o cálculo do índice, entre outras funções. Ambos foram coletados após $24 \mathrm{~h}$ de permanência do paciente na UTI. Os dados obtidos foram inseridos em planilha eletrônica do programa Microsoft Office Excel e apresentados em forma de mediana, média \pm DP e percentagens. Os testes Qui-quadrado e coeficiente de Pearson foram utilizados para comparação das proporções. O nível significativo adotado foi de $5 \%$. Para os cálculos utilizou-se o EPI INFO-6.

\section{RESULTADOS}

Do total de pacientes estudados (148), houve predomínio do sexo masculino $(59,4 \%)$. A idade média foi de $54,3 \pm 16,1$ anos com mediana de 55,5 anos. Com relação à faixa etária, encontrou-se maior número de pacientes com idade entre 46 e 60 anos (34,5\%), seguido de 61 a 75 anos $(28,4 \%)$ e 31 a 45 anos $(20,2 \%)$. A minoria foi representada pelos pacientes com idade inferior a 18 anos (1,3\%). A maioria dos pacientes procedia do pronto-socorro (73,6\%), seguida do centro-cirúrgico $(22,3 \%)$. As clínicas médica e cirúrgica perfizeram $3,4 \%$ e somente um paciente foi proveniente de outro hospital. O tempo médio de permanência na UTI foi de $9,1 \pm 10$ dias. As doenças do aparelho circulatório representaram o principal motivo de internação na UTI em 56 pacientes, seguidas das doenças dos aparelhos digestivo e respiratório (22 cada). Durante a internação na UTI, 29,7\% dos pacientes evoluíram para óbito. O escore médio do NAS foi de $51,5 \pm 12,1$ e do APACHE II foi 13,4 $\pm 8,3$ (Tabela 1). Ao associar os valores do NAS com o APACHE II, identificou-se uma curva ascendente com valor de $R=0,82$, demonstrando correlação positiva entre eles (Figura 1). Separando os pacientes com valores de NAS > 51 observou-se que 41/83 não sobreviveram e 42/83 sobreviveram; já naqueles com NAS < 51,3/65 não sobreviveram e 62/65 sobreviveram $(p<0,005)$, identificando-se diferença estatística significativa entre os grupos (Tabela 2).

Tabela 1 - Valores Médios Segundo NAS e APACHE II

\begin{tabular}{lc}
\hline & Média \pm DP \\
\hline NAS & $51,5 \pm 12,1$ \\
APACHE II & $13,4 \pm 8,3$ \\
\hline
\end{tabular}

Tabela 2 - Taxa de Mortalidade Segundo Valores do NAS

\begin{tabular}{lccc}
\hline & $\begin{array}{c}\text { Não } \\
\text { Sobreviveram }\end{array}$ & Sobreviveram & Total \\
\hline $\begin{array}{l}\text { NAS acima } \\
\text { de 51 }\end{array}$ & $41(49,3 \%)$ & $42(50,7 \%)$ & $83(100 \%)$ \\
$\begin{array}{l}\text { NAS abaixo } \\
\text { de 51 }\end{array}$ & $3(4,6 \%)$ & $62(95,4 \%)$ & $65(100 \%)$ \\
\hline
\end{tabular}

$<0,005$

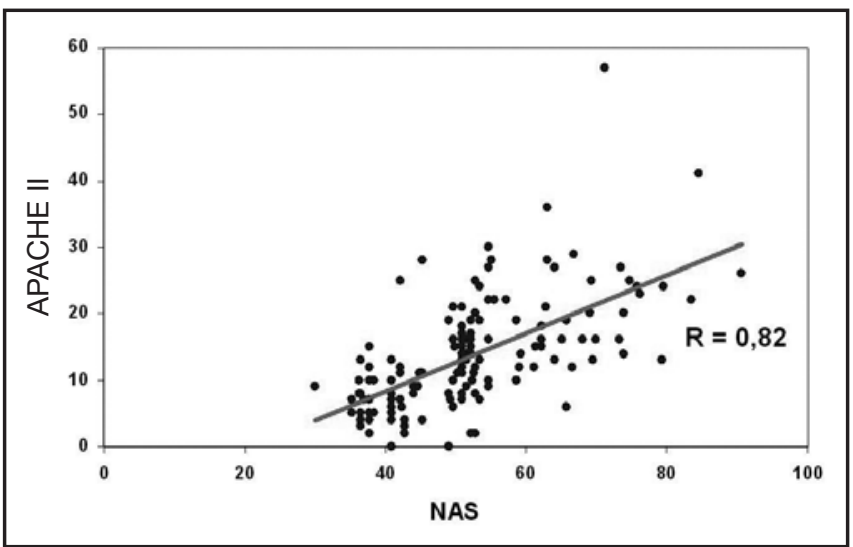

Figura 1 - Correlação de Valores de NAS e APACHE II

\section{DISCUSSÃO}

Os índices de gravidade estão cada vez mais difundidos e utilizados em unidades de terapia intensiva, permitindo tanto a avaliação do desempenho da unidade, quanto a eficiência do tratamento utilizado ${ }^{1}$. Diferentemente encontra-se a avaliação da carga de trabalho de Enfermagem, onde ocorre menor proporção de enfoque, porém crescente interesse e preocupação em estudá-la para possibilitar a avaliação da complexidade do paciente, objetivando constituir um instrumento facilitador no real dimensionamento do pessoal da enfermagem, visando melhorar a qualidade da assistência. Existe uma percepção entre os profissionais da saúde que os pacientes mais graves demandam maior carga de trabalho de enfermagem, mas não foram encontrados na literatura estudos que confirmem tal hipótese. 
Observou-se neste estudo tal preocupação, através da correlação de um índice de gravidade mundialmente conhecido e utilizado, o APACHE II, com instrumento que mede a carga de trabalho de enfermagem, o NAS, índice mais recentemente publicado, com grande interesse pela comunidade científica, por representar a medida da real carga de trabalho de enfermagem.

$\mathrm{Na}$ caracterização dos pacientes atendidos neste estudo, observaram-se algumas peculiaridades da unidade, por ser uma UTI que atende diferentes especialidades. Quando abordado o tempo de permanência, identificou-se o resultado de 9,1 \pm 10 dias, valor considerado superior aos estudos de Queijo: 4,6 $\operatorname{dias}^{7}$ e Bernat e col.: 4,3 $\pm 5,4$ dias $^{8}$.

As doenças do aparelho circulatório representaram o principal motivo de internação na UTI em 56 pacientes, seguidas das doenças dos aparelhos digestivo e respiratório (22 cada). Esses dados acompanham as estatísticas do DATASUS que, no mesmo período do estudo, confirmaram as doenças do aparelho circulatório, seguidas das doenças dos aparelhos digestivo e respiratório como as principais causas de internação hospitalar9.

O escore médio do NAS foi de $51,5 \pm 12,1$. A média do NAS obtida não se assemelha aos valores encontrados na literatura, sendo inferior ao estudo de Queijo7: 67,1 $\pm 8,42$ e superior ao de Bernat e col. $.^{8}: 40,8 \pm 14,1$.

Através do coeficiente de Pearson, identificou-se neste estudo a correlação positiva entre o APACHE II e o NAS $(R=0,82)$. Nenhum estudo na literatura utilizou o APACHE II na comparação com o NAS. Queijo ${ }^{7}$ testou a hipótese de que quanto mais grave o paciente maior é a demanda de trabalho de enfermagem utilizando um outro índice de gravidade, o SAPS II. Ao correlacionálos através do coeficiente de Pearson, Queijo ${ }^{7}$ identificou baixa correlação linear entre o NAS e o SAPS II. Durante a internação na UTI, 29,7\% dos pacientes evoluíram para óbito. Este resultado difere de Queijo7, em que a taxa de mortalidade foi de 17,5\%. Em seu estudo $28 \%$ dos pacientes eram neurológicos. Os escores do APACHE II e do SAPS II são muito influenciados pela escala de coma de Glasgow. Interessante notar que a mortalidade observada ficou muito aquém daquela calculada pelo SAPS II. É possível que o peso das escalas de coma superestime o risco de óbito, mas certamente não o fazem com a carga de trabalho.

Os dados deste estudo levaram às seguintes conclusões: o valor médio do NAS foi de $51,5 \%$, o APACHE II apresentou correlação positiva com o NAS $(R=0,82) \mathrm{e}$ identificou-se que a mortalidade é maior nos pacientes com NAS acima da média.

Com base nestes resultados, faz-se necessário realizar novos estudos, com grupos de pacientes admitidos em diferentes UTI, seja geral ou específica (trauma, pósoperatório, neurológica, etc.) e com amostras maiores, para que se possa consolidar a verdadeira correlação entre gravidade do paciente e carga de trabalho de enfermagem.

\section{REFERÊNCIAS}

01. Livianu J, Anção MS, Andrei AM et al - Índices de Gravidade em UTI - Adulto e Pediátrica, em: Knobel E - Condutas no Paciente Grave. $2^{a}$ Ed, São Paulo: Atheneu, 1999;1334-1352.

02. Knaus WA, Zimmerman JE, Wagner DP et al - APACHE-acute physiology and chronic health evaluation: a physiologically based classification system. Crit Care Med, 1981;9:591-597.

03. Knaus WA, Draper EA, Wagner DP et al - APACHE II: a severity of disease classification system. Crit Care Med, 1985;13:818-829.

04. Cullen DJ, Civetta JM, Briggs BA et al - Therapeutic intervention scoring system: a method for quantitative comparison of patient care. Crit Care Med, 1974;2:57-60.

05. Miranda DR, de Rijk A, Schaufeli W - Simplified Therapeutic Intervention Scoring System: the TISS-28 items-results from a multicenter study. Crit Care Med, 1996;24:64-73.

06. Miranda DR, Nap R, de Rijk A de et al - Nursing activities score. Crit Care Med, 2003;31:374-382.

07. Queijo AF - Tradução para o português e validação de um instrumento de medida de carga de trabalho de enfermagem em Unidade de Terapia Intensiva: Nursing Activities Score (NAS). São Paulo: Escola de Enfermagem da USP; 2002.

08. Bernat Adell A, Abizanda Campos R, Cubedo Rey M et al - Nursing Activity Score (NAS). Our experience with a nursing load calculation system based on times. Enferm Intensiva, 2005;16:164-173.

09. Brasil. Ministério da Saúde. Estatísticas de morbidade. Internação hospitalar do SUS segundo capítulo do CID-10. [on line]. Brasília (DF): Ministério da Saúde; 2005 [citado 2006 maio 9]. Disponível em: http://tabnet.datasus.gov.br/cgi/tabcgi.exe?sih/cnv/miuf.def. 